PAULETTE DIETERLEN

\title{
Teoría de la elección racional, un ejemplo. del individualismo metodológico
}

0

UIZA sea un lugar común identificar el nacimiento del Individualismo Metodológico con la obra de Hobbes. Sin embargo creo que es conveniente recordar que con su Leviathan se inicia una nueva forma de explicar los fenómenos políticos, sociales y económicos. Hobbes nos heredó dos ideas: 1) que los hombres actúan por interés propio; 2) que las acciones colectivas deben de explicarse por las motivaciones individuales de las personas que forman parte de la sociedad.

La prioridad del fenómeno individual sobre el colectivo para explicar cualquier clase de acciones ha sido sostenida por un sinnúmero de pensadores desde Hobbes hasta nuestros días. Pienso en Hume, en Mill, en ocasiones Marx' ${ }^{1}$, Weber y en la actualidad por varios filósofos de las ciencias sociales y por varios economistas, la mayoría de ellos formados dentro de la corriente denominada "neoclásica".

Si bien he caracterizado al individualismo metodológico de una determinada forma, como la explicación de cualquier acción por las motivaciones individuales, estoy consciente de que se ha aplicado e interpretado de varias formas por lo que me referiré en este trabajo a la obra de J. Elster, y tomaré exclusivamente una de sus vertientes: la teoría de la elección racional.

También explicaré una aplicación de la teoría de la elección racional, la de la Teoría del Capital Humano.

1 En Marx existen varios tipos de explicaciones sobre los fenómenos sociales. Sin embargo, Jon Elster insiste en la importancia que tiene el individualismo metodológico en la obra de Marx. Véase: Jon Elster. Making Sense of Marx. Cambridge University Press. Cambridge 1985 pp. 5-28. 
Por último mencionaré algunas dificultades que presenta la teoría de la elección racional y algunos puntos a favor de ella.

\section{El individualismo metodológico según J. Elster ${ }^{2}$}

El individualismo metodológico es la doctrina que sostiene que todos los fenómenos sociales - su estructura y sus cambios - son en principio explicables por elementos individuales, es decir por las propiedades de los individuos tales como sus metas, sus creencias y sus acciones. Obviamente para lograr esta clase de explicaciones tenemos que llevar a cabo cierto reduccionismo. Si pasamos del estudio de una institución social o de ciertos patrones de conducta agregativa, realizamos la misma actividad que cuando pasamos, por ejemplo, del estudio de las células a las moléculas.

$\mathrm{La}$ racionalidad del reduccionismo se explica de la siguiente manera: como la meta de la ciencia es explicar por medio de leyes, es necesario reducir tanto como sea posible, el "lapso de tiempo" entre el "explanans" y el "explanandum", entre la causa y el efecto con el objeto de evitar explicaciones espúreas.

Una explicación espúrea puede darse por dos razones: 1) cuando una variable, no tomada en consideración, genera tanto la causa aparente como el efecto aparente; 2) cuando el efecto surge por una causa que toma el lugar de la causa que aparece en la ley.

Los riesgos se reducen si logramos establecer una cadena continua de causas y efectos, esto es cuando reducimos el lapso de tiempo entre el explanans y el explanandum.

El reduccionismo, considerado bajo esta perspectiva, no es un fin en sí mismo sino un medio tanto para dar una explicación más detallada, como para lograr comprender mejor qué sucede cuand o pasamos de lo macro a lo micro y de lapsos de tiempo más breves a más largos.

Explicar, según esta versión del individualismo metodológico, es poseer un mecanismo para abrir la "caja negra" y mostrar las tuercas, los cerrojos, los eslabones, los engranajes, los deseos y las creencias que generan las acciones cuyas conse- 
cuencias van a incorporarse en un patrón agregativo.

Elster caracteriza al individualismo metodológico de la siguiente manera:

1. No presupone el egoísmo, ni siquiera la racionalidad de las acciones individuales.

Para explicar la conducta individual se parte de esas características pero como una consideración metodológica y no como una asunción acerca de la naturaleza humana. Es más, actualmente existe una amplia bibliografía sobre la relación entre el altruismo y la racionalidad ya que el egoísmo no explica ni la racionalidad de las acciones colectivas, tal y como lo demuestra el Dilema del Prisionero, ni la participación de los agentes para la obtención de los bienes públicos, tal como lo demuestra el problema del gorrón. ${ }^{3}$

2. El individualismo metodológico sólo se sostiene en contextos extencionales. Cuando las entidades agregadas aparecen en contextos intencionales no pueden ser reducidas a entidades individuales. Las personas a veces tienen creencias acerca de entidades supraindividuales que no pueden ser reducidas a creencias individuales. Por ejemplo, no podemos reducir la proposición: "El equipo tricolor tiene mucho corazón y en la cancha lo demostrará" a los deseos de vencer que tiene cada uno de los jugadores.

Podemos decir, a grosso modo, que un contexto extencional es aquél en el que se da un intercambio en las variables "salva veritate"4, lo que significa que podemos sustituir unas variables por otras y la relación entre ellas sigue siendo verdadera.

3. Existen ciertas propiedades en los individuos como por ejemplo "el ser poderoso" expresados por términos relacionales de tal manera que una descripción cuidadosa de esta característica en un individuo nos remite necesariamente a otro.

4. La convivencia de la reducción no debe de cegarnos

${ }_{3}$ Dos posibilidades de explicar la racionalidad del altruismo se encuentran en: Thomas Nagel. The Possibility of altruism. Princeton University Press. 1978. Y en: Amartya Sen. Rational Fools. Philosophy and Economic Theory. Hahn y Hollis (Eds.) Oxford University Press. Oxford 1979 pp. 87-109.

4 Para un estudio de la diferencia entre contex tos intencionales y extencionales, véase: W.V.O. Quine. Two Dogmas of Empiricism. En: From a Logical Point of View. Harvard University Press. Cambridge. 1980 pp. 20-46. 
acerca del peligro de un reduccionismo prematuro.

Existe el peligro de querer explicar los fenómenos sociales complejos en términos de motivaciones y creencias individuales, lo que puede originar explicaciones arbitrarias.

Ahora bien, es necesario comentar que el individualismo metodológico utiliza las explicaciones intencionales para dar cuenta de los fenómenos individuales. El explananda de las explicaciones intencionales está formado por las acciones individuales de los agentes. Una acción colectiva puede ser explicada si distribuimos los propósitos y las metas del grupo en cada uno de los agentes. Lo importante de las explicaciones intencionales es la especificación de la meta, es decir la especificación del estado de cosas que el grupo desea y por el que la acción se lleva a cabo.

Las explicaciones intencionales más desarrolladas son las que da la teoría de la elección racional. ${ }^{5}$

\section{La Teoria de la Elección Racional.}

Según Elster ${ }^{6}$ la teoría de la elección racional es una teoría que puede ser vista desde dos ángulos, como una teoría normativa y como una teoría descriptiva.

Como teoría normativa, la teoría de la elección racional nos dice qué es lo que debemos de hacer para lograr ciertas metas lo mejor posible aún cuando no nos dice cuáles deben de ser esas metas.

Podemos afirmar que esta teoría ofrece imperativos hipotéticos y no categóricos.

Como teoría descriptiva, su misión es la de ayudarnos a predecir ciertas acciones.

La teoría de la elección racional para justificar y explicar una conducta recurre a tres elementos necesarios para garantizar que se dé una situación de elección.

1) Un grupo de cursos de acción factibles que satisfagan

5 Me parece que es más correcto hablar de elección racional más que de preferencias reveladas porque desde el punto de vista del investigador, primero se observan las elecciones y de ahí se infieren las preferencias; para ampliar este punto véase: Amartya Sen. Behavior and the concept of preference. En: Rational Choice. Jon Elster (Ed.). Blackwell. Oxford 1986 pp. 60-81.

6 Véase. Jon Elster. Rational Choice. Introduction. op. cit. pp. 1-33. 
ciertas restricciones lógicas, físicas y económicas.

2) Un grupo de creencias racionales sobre la estructura causal de la situación que va a determinar qué cursos de acción. nos llevan a qué resultados.

3) Un ordenamiento subjetivo de làs alternativas factibles que se derivan delordenamiento de las consecuencias a las que se quiere llegar.

Actuar racionalmente significa elegir la alternativa con la jerarquía más alta dentro de un grupo de alternativas factibles.

Ahora bien, la situación de elección se divide principalmente en dos dimensiones:

1) Es necesario hacer una distinción entre información perfecta e imperfecta. Las situaciones en donde tenemos una información perfecta acerca de todos los cursos de acción alternativos son muy difíciles de darse, por esta razón son más importantes las situaciones de elección en donde la información es imperfecta. De este hecho se derivan dos situaciones: el riesgo y la incertidumbre.

El riesgo se define como una situación en la que se adscriben a las consecuencias de ciertos cursos de acción ciertas probabilidades numéricas. La incertidumbre es una situación en la que el criterio normativo para tomar una decisión consiste en elegir la opción que maximice la utilidad esperada.

2) Otra distinción importante que merece la pena tomarse en cuenta en una situación propia de elección racional es la que existe entre decisiones paramétricas y decisiones estratégicas.

Una decisión es paramétrica cuando el agente enfrenta restricciones externas que ya están dadas. El agente primero estima las restricciones y luego decide qué hacer.

Por el contrario en una situación estratégica se da una interdependencia entre las decisiones de distintos agentes.

$\mathrm{El}$ agente, antes de tomar su decisión, tiene que preveer qué es lo que los otros van a hacer, y tiene que preveer lo que los otros van a pensar que va a hacer él.

Podríamos pensar que esta situación da origen a un regreso al infinito pero esto no es así puesto que se puede llegar a un punto de equilibrio. Dicho punto se logra cuando se dan las siguientes características:

a) Existe un grupo de decisiones por tomar; b) cada una de las personas que está actuando toma una decisión; c) la decisión tomada por una persona afecta a las decisiones que los 
otros tomen; d) una vez que cada persona toma su decisión ninguno puede mejorar su situación si se desvía de la situación de equilibrio, siempre y cuando los otros mantengan su decisión.

Las situaciones estratégicas son el tópico de la teoría de los juegos, la cuál es un elemento indispensable para la teoría de la elección racional.

La teoría de juegos contiene los elementos siguientes: hay un grupo de agentes y para cada uno de ellos existe un grupo de elecciones y de estrategias posibles. Cuando los agentes eligen un elemento de la estrategia, originan un cierto estado de cosas. Cada uno de los agentes tiene una preferencia jerarquizada por un posible estado del mundo.

En las situaciones estratégicas pueden darse tres clases de interdependencia:

1) La recompensa de cada uno de los agentes depende de la recompensa de todos; 2) la recompensa de cada uno de los agentes depende de la elección de todos; 3) la elección de.cada agente depende de la elección de todos.

La teoría de juegos estudia estas situaciones desde dos perspectivas. Primero, la perspectiva de la no cooperación que considera el problema de las decisiones desde el punto de vista de la racionalidad individual. El problema en este caso es que una serie de elecciones individuales racionales puede llevar a resultados para el propio agente peores que si hubiese escogido otra estrategia. Segundo, la perspectiva de la cooperación que asume que esto no sucederá.

Ahora bien, los juegos no cooperativos pueden dividirse tanto por su estructura como por sus resultados.

Primero existe una diferencia entre un juego de suma constante y uno de suma variable. Mientras que en el primer caso hay un total que se divide entre los agentes de tal manera que si uno gana el otro pierde, en el segundo caso - en el caso de suma variable - ambos agentes pueden perder o ganar. Mientras que los juegos de suma constante son juegos de conflicto, los de suma variable pueden ser de cooperación, o de estrategia mixta, es decir, de conflicto y de cooperación.

Estos últimos juegos forman el tópico central de la teoría de los juegos, ejemplo de esto son "La Batalla de los Sexos", "El Dilema del Prisionero" y "El Juego de la Seguridad".

Un elemento que, según $\mathbf{J}$. Elster, hạ sido subestimado 
por la teoría de los juegos es el tiempo. Según él las situaciones de elección en las que el tiempo juega un papel importante son extrañas pues las consecuencias de cualquier acción están dispersas en el tiempo. Una alternativa puede darnos beneficios mayores en un tiempo posterior.

Para poder elegir entre alternativas cuyas consecuencias tienen patrones temporales distintos es necesario tener una manera de justificar racionalmente el peso de los beneficios en momentos distintos del tiempo.

Pasemos ahora a examinar la estructura de la explicación de la elección racional. Como vimos con anterioridad, la explicación de la elección racional es una subclase de explicaciones intencionales. Dichas explicaciones se caracterizan por la relación que existe entre la acción que va a ser explicada y los deseos y las creencias de los agentes.

La relación se define por tres condiciones:

1) Los deseos y las creencias del agente son razones para la conducta;

2) Los deseos y las creencias causan la cónducta;

3) Los deseos y las creencias causan la conducta en tanto que razones.

Ahora bien, estas condiciones son necesarias para cualquier explicación intencional. Una explicación de elección racional necesita dos condiciones de consistencia, tanto las creencias como los deseos pueden ser internamente consistentes.

También se requiere que las creencias y los deseos estén fundamentados en una evidencia que se encuentra a la mano.

La condición de la evidencia se divide en tres:

1) La creencia necesita de un grado máximo de plausibilidad inductiva, dada la evidencia.

2) La creencia es causada por la evidencia que se encuentra a la mano.

3) La evidencia causa la creencia de la manera correcta.

Es conveniente imponer una condición de optimalidad sobre la evidencia que puede recolectarse antes de que se forme una creencia. Una decisión de actuar puede estar rodeada de un área de penumbra, lo importante en este caso es tener un criterio para distinguir cuándo es racional recolectar cierta información y cuándo no tienen caso recolectarla.

La estructura de la racionalidad está constituida por una 
serie de creencias y de deseos que no sólo explican la acción sino que tienen una cadena causal que puede ser identificada. Esto se logra cuando se cumplen tres condiciones de optimalidad: 1) la acción es la mejor manera que tiene el agente para satisfacer sus deseos dadas sus creencias; 2) la creencia es lo que mejor puede formarse dada la evidencia; 3) el monto de la evidencia que se ha recogido es óptima dado su deseo.

Podemos decir resumidamente que estos elementos son indispensables para poder hablar de una elección racional.

\section{Un ejemplo en Economia: "La Teoría del Capital Humano"}

La teoría del capital humano según Mark Blaug7 fue anunciada hace más de veinte años por Theodore Schultz. Sin embargo podemos decir que su nacimiento efectivo se produjo dos años más tarde cuando el Journal of Political Economy en 1962, publicó un suplemento sobre "La Inversión en Seres Humanos". Este volumen incluía, entre otros, los capítulos preliminares de la monografía "Human Capital"s publicada en 1964 por Gary Becker que desde entonces se ha convertido en un punto obligado de referencia en este tema.

El punto medular de la teoría del capital humano consiste en pensar que las personas gastan en sí mismas de formas diversas y que no sólo buscan una satisfacción presente sino también un rendimiento en el futuro, sea este pecunario o no pecunario.

Las personas adquieren, por ejemplo, cuidados sanitarios, compran voluntariamente educación, gastan tiempo buscando empleo, compran información acerca de las oportunidades del empleo, emigran buscando mejores oportunidades de empleo, etc. Estos fenómenos pueden ser considerados como gastos de inversión más que como gastos de consumo, independientemente de que las realicen los individuos en beneficio propio o de que las realice la sociedad en beneficio de sus miembros.

Lo que une a los fenómenos vistos anteriormente es que sea quien sea el agente que tome las decisiones, éste está

${ }^{7}$ Mark Blaug. La Metodología de la Economía. Alianza Universidad Madrid. 1985 p. 250.

${ }^{8}$ Gary Becker. Human Capital. Columbia University Press. New York. 1964. 
considerando al futuro como una justificación racional para llevar a cabo las acciones presentes.

Quizá donde aparece el planteamiento de Becker con más claridad es en el artículo llamado "The Economic Approach to Human Behavior"9

Según Becker, lo que distingue a la economía de otras ciencias sociales no es tanto el objeto propio sino su acercamiento.

La característica específica del acercamiento económico radica en asumir que, un gobierno, un individuo, una firma o un sindicato asume explícita y extensivamente una conducta maximizadora con el objeto de lograr más utilidad o una función de bienestar mayor.

El acercamiento económico asume, también, la existencia de mercados que eficientemente coordinan las acciones de los participantes de tal manera que su conducta sea consistente.

Según Becker como los economistas no tienen mucho que decir acerca de la formación de preferencias se asume que éstas no cambian ni con el tiempo, ni entre las personas, da igual que estas sean pobres o ricas o que pertenezcan a culturas o sociedades distintas.

Con esta tesis Becker elimina el problema de buscar una justificación para preferir una acción o un estado de cosas que se da en un futuro remoto más que en uno próximo.

Sin embargo, la teoría del Capital Humano contiene todos los elementos de la teoría de la elección racional. Los precios y otros instrumentos de mercado distribuyen los recursos escasos de una sociedad mostrando qué es lo que es factible elegir, permitiendo que las personas formen ciertas creencias y restrinjan sus deseos.

Ahora bien, las preferencias que determinan las elecciones no se refieren exclusivamente a bienes y servicios de mercados tales como manzanas, automóviles y cuidados médicos, sino a los objetos de elección que estos bienes y servicios representan y que son producidos por cada uno de los agentes.

Los objetos de elección, que dichos bienes representan, están relacionados con aspectos fundamentales de la vida tal como el placer sensual, la vida, la benevolencia, la envidia, etc.

${ }^{9}$ Gary Becker. The Economic approach to human behavior. En: Rational Choice. op. cit. pp. 108-122. 
y no siempre tienen una relación estable con los bienes y servicios del mercado.

El núcleo del programa de investigación de la Teoría del Capital Humano está formado por la conducta maximizadora, por el equilibrio del mercado, que nos da información y por la asunción de la estabilidad de las preferencias.

Según Becker el acercamiento económico no se restringe a los bienes materiales ni, exclusivamente, al sector del mercado. Los precios pueden referirse al "dinero" si hablamos del mercado y a la "sombra" si hablamos fuera del mercado. Estos to precios miden el costo de oportunidad en el uso de los recursos escasos.

El acercamiento económico no asume que todos los participantes en el mercado, tienen necesariamente una información completa o que realizan transacciones sin costo alguno. Es importante distinguir entre una transacción costosa y una conducta irracional. De acuerdo con Becker y con cualquier teórico de la elección racional, es necesario saber cuánd o ya no es posible seguir recogiendo información. Por eso es necesario desarrollar una teoría de la acumulación óptima o racional de la información costosa, teoría que contemple la idea de invertir más cuando se trata de obtener una decisión "mayor". Por ejemplo, probablemente necesitamos más información si tenemos que decidir si nos casamos o no o con quien, que si tenemos que decidir si compramos o no una casa.

La teoría del Capital Humano no asume, por supuesto, que los agentes que toman las decisiones son necesariamente conscientes del esfuerzo por maximizar, tampoco asume que los agentes puedan verbalizar o describir de una manera precisa las razones que tienen para actuar de una manera determinada. Sólo asume que el acercamiento económico es comprehensivo y aplicable a toda la conducta humana, ya sea que dicha conducta se refiera a precios en el mercado, a precios "sombra", a decisiones que se repiten o que son poco frecuentes, a decisiones pequeñas o grandes, a fines emotivos o económicos, a decisiones tomadas por personas pobres o ricas, por hombres o por mujeres, por adultos o por niños, etc.

Quizá uno de los ejemplos más representativos de la Teoría del Capital Humano, se encuentra en la explicación que 
da Becker del matrimonio y del divorcio ${ }^{10}$. De acuerdo con este acercamiento, una persona decide casarse cuando la utilidad esperada del matrimonio excede a la utilidad de quedarse soltero o a la utilidad de buscar una pareja mejor. Así, una personà casada termina su matrimonio cuando la utilidad anticipada de regresar a la soltería, o de casarse con otra persona, excede a la pérdida de utilidad de la separación, incluyendo las pérdidas que se dan por asuntos como la separación física de los hijos, por el pago de cuotas legales, etc. ya que muchas personas buscan parejas, el mercado de matrimonios puede existir.

Becker afirma que la conducta humana no está dividida en compartimientos de tal manera que algunas veces se explique como capaz de maximizar y otras como incapaz de hacerlo; que a veces se explique como motivada por preferencias estables y a veces por preferencias volátiles; a veces como resultado de una acumulación óptima de información y a veces como carente de esa información. Toda la conducta humana puede ser vista como un grupo de acciones que se explican, por los deseos y las creencias de los participantes, por la maximización de un grupo estable de preferencias y por la acumulación "racional" de información.

Según Becker, si el argumento central de la teoría del Capital Humano es correcto, el acercamiento económico provee una red para comprender la conducta humana, tal y como lo desearon Bentham, Comte y Marx.

Imaginemos una explicación del juicio de Paris a la manera de Gary Becker.

Hallándose los dioses reunidos para celebrar la boda de Tertis y Peleo, Eride (la discordia) arrojó en medio de ellos una manzana de oro con la consigna de que debía ser otorgada a la más hermosa de las tres diosas: Atenea, Hera y Afrodita. Debido a que no hubo acuerdo entre los dioses, pues ninguno quería pronunciarse en favor de una de las tres divinidades, Zeus encargó a Hermes que llevase a Hera, a Atenea y a Afrodita al monte Ida para que Paris fallase el pleito. Las tres diosas defendieron su causa ante él, prometiéndole tanto su protección, como ciertos dones si fallaba en su favor. Hera se comprometió a darle el imperio de Asia; Atenea le ofreció la

10 Ibid. p. 115. 
virtud de la prudencia y le aseguró la victoria en todos los combates; Afrodita se limitó a ofrecerle el amor de Elena, la mujer más bella de la tierra. Paris decidió que Afrodita era la más hermosa después de haber hecho el siguiente cálculo: si, en principio, las tres ofrecen la misma utilidad, tras un momento de reflexión, Paris puede darse cuenta de que el valor de Atenea se reduce ya que no hay ninguna garantía de que cumpla su promesa. La utilidad de Hera es mayor porque enemistarse con ella es muy costoso; sin embargo, a Paris no le es atractivo el ser el dueño de Asia. Seguramente Afrodita es la que más utilidades conservó aún después de haber descontado el costo del enojo de Hera y de Atenea. Por eso fue la elegida.

En este ejemplo vemos muy claramente la relevancia de la información. Probablemente Paris al realizar su elección ignoraba que Elena era una mujer casada y que su amor por ella desencadenaría la guerra de Troya. ${ }^{11}$

\section{Los límites de la Teoría de la Elección Racional}

Confieso que este ejemplo ha sido un poco perverso ya que la teoría de la elección racional tiene aplicaciones más interesantes. El problema con Becker es que no se enfrenta con problemas a los que otros teóricos de la elección racional dan respuesta. Por ejemplo la inclusión del tiempo, los cambios de las preferencias, el origen de las preferencias, etc.

Elster, con un espiritu menos triunfalista que Becker analiza las limitaciones posibles de la teoría. Ve que pueden surgir dos clases de problemas: 1) las funciones explicativas y normativas de la teoría, presuponen que la noción de elección racional está bien definida, si no somos capaces de explicar lo que es una elección racional, la teoría falla; 2) aún cuando podamos explicar lo que es una elección racional, la teoría no cumple con su función explicativa si la conducta observada es diferente a la conducta normativa prescrita.

El primer problema puede surgir tanto por la inexistencia como por la carencia de unicidad en las acciones, las creencias y la evidencia. El primer caso está representado por el "asno de Buridán". Hay deseos y creencias fijas pero hay más de una

1 Véase el desarrollo de la historia en: Martín Hollis. Rational Preferences. the Philosophical Forum. vol. XIV, No 3-4 Spring-Summer 1983 pp. 246-262. 
acción prescrita por la teoría de la elección racional, razón por la cual puede surgir la indiferencia. Así el asno de Buridán muere de hambre porque no puede elegir entre dos montones de paja. No hay conducta racional si el agente no es capaz de comparar pares de alternativas.

La inexistencia de una creencia racional surge cuando realizamos una elección en una situación de incertidumbre.

Por último, la inexistencia de un grado óptimo de evidencia surge cuando es imposible encontrar un valor marginal al costo que representa tener información.

Una segunda crítica a la teoría de la elección racional afirma que aún cuando las personas sean racionales en algunas ocasiones, en otras no lo son. La racionalidad puede violarse en cada eslabón de la cadena explicativa: en la acción, en la formación de creencias y deseos, y en la acumulación de la evidencia.

En el nivel de la acción, la irracionalidad se manifiesta en casos como el de la debilidad de la voluntad, en la búsqueda de utilidad no esperada, en formas de acción autodestructivas, etc. 12

En el nivel de formación de creencias, hay evidencia de que las creencias están influenciadas por formas de irracionalidad "frías" y "calientes". Encontramos casos de irracionalidad caliente o motivada en lo que se ha llamado (y es imposible de traducir) "wishful thinking", es decir cuando el deseo determina la creencia. El ejemplo perfecto es el de la fábula de la zorra que no pudiendo alcanzar las uvas, decide que no las quiere porque están verdes. La irracionalidad "fría" o cognitiva en la formación de creencias se muestra en casos como el de realizar generalizaciones a partir de pocos casos, y en casos como tratar probabilidades como si fueran sumas.

Por otra parte, un caso de irracionalidad en el nivel de la recolección de evidencia surge cuando la cantidad de información recolectada está por debajo o por encima de lo que debería estar.

Otra duda que surge acerca del poder explicativo y normativo es la de si realmente las ciencias sociales pueden partir del hecho de que los hombres "toman decisiones"cada vez que

12 Para un estudio de ciertas formas de irracionalidad ver: Jon Elster. Sour Grapes. Cambridge University. Press. Cambridge 1985. 
se encuentran delante de una alternativa. Una alternativa podría ser explicar las acciones humanas en términos de normas sociales más que de elecciones individuales ${ }^{13}$. Los seres humanos realizamos acciones por hábito, por tradición, por costumbre, por un deber. En estos casos, las normas sociales serían, en el orden explicativo, anteriores a las decisiones individuales.

Sin minimizar estos problemas, Elster sigue defendiendo el papel normativo y el explicativo de la teoría de la elección racional, por ejemplo, para detectar formas de irracionalidad necesitamos una caracterización de la racionalidad. Por otra parte, afirmar que muchas veces actuamos de acuerdo con normas, hábitos o deberes no va en contra de la teoría ya que puede uno elegir seguir una norma o violarla. La teoría no asume que toda nuestra conducta se ajusta automáticamente a las oportunidades inmediatas. Podemos elegir ciertas normas de cooperación, por ejemplo, en contra de normas de beneficio inmediato.

Yo estoy consciente de las limitaciones del individualismo metodológico; sin embargo, pienso que ha sido subestimado. Creo que tomando en cuenta los problemas que presenta, y evitando caer en el absurdo de rechazarlo porque no explica, por ejemplo, porque escribió Baudelaire las Flores del Mal, el individualismo metodológico puede ser de mucha utilidad para las ciencias sociales. Esto es, claro está si seguimos otorgándole algún valor a la razón llamada instrumental.

13 Para una discusión sobre la diferencia entre actuar siguiendo una regla y por egoísmo, véase: Martín Hollis. Rational mand and social science. en: Rational Action. Ross Harrison (Ed.). Cambridge University Press. Cambridge 1979 pp. 1-16. 